\section{New molecular targets for the treatment of neovascular age-related macular degeneration}

\section{Gian Marco Tosi, ${ }^{1}$ Marcella Barbarino, ${ }^{2}$ Maurizio Orlandini, ${ }^{3}$ Federico Galvagni ${ }^{3}$}

1Ophthalmology Unit of the Department of Medicine, Surgery and Neuroscience, University of Siena; ${ }^{2}$ Department of Medicine, Surgery and Neuroscience, University of Siena, Siena; ${ }^{3}$ Department of Biotechnology, Chemistry and Pharmacy, University of Siena, Siena, Italy

\begin{abstract}
Age-related macular degeneration (AMD) is a progressive chronic disease that currently represents the leading cause of irreversible vision loss in the western world. Experimental and clinical evidence has demonstrated that vascular endothelial growth factor A (VEGF-A) plays an important role in promoting the choroidal neovascularization that characterizes the wet form of AMD. Intravitreal injection of antiVEGF-A agents is the current treatment of choice for neovascular AMD (nAMD). These agents have brought about dramatic changes in the treatment of nAMD, but most patients require frequently repeated injections and regular long-term follow-up, with a significant percentage of them showing resistance to anti-VEGF-A drugs. Thus, the identification of additional therapies that could improve the treatment protocols is needed. There are numerous areas of investigation into new treatments, with increasing efforts being made to study drugs that address various targets along the angiogenic signaling cascade, or other pathways related to the onset of nAMD. The aim of the present review is to summarize and discuss promising new therapies and targets that have the potential to improve outcomes and to lengthen treatment durability, especially in patients with recalcitrant or recurrent forms of nAMD.
\end{abstract}

\section{Introduction}

Age-related macular degeneration (AMD) is the leading cause of blindness in developed countries and its prevalence and incidence is likely to increase dramatically with the aging of the population. ${ }^{1}$ The exudative form of AMD, although less common than the atrophic form, is more threatening to vision: $90 \%$ of those with severe visual loss due to AMD suffer from the exudative type.

The main pathological feature that characterizes the exudative form of AMD is choroidal neovascularization (CNV). Although many aspects of the molecular pathogenesis of exudative AMD have not yet been elucidated, experimental and clinical evidence has demonstrated that vascular endothelial growth factor A (VEGF-A) plays a central role in promoting $\mathrm{CNV}^{2-4}$ Intravitreal injection of anti-VEGF-A agents is the current treatment of choice for neovascular AMD (nAMD), and five molecules have been introduced into clinical practice since 2004 (bevacizumab, pegaptanib, ranibizumab, aflibercept and a molecule newly approved for the Chinese market, conbercept). This has brought about dramatic changes in the management of the pathology, but the challenges related to the treatment of nAMD remain numerous. Thus, there is a need for efforts to investigate new areas involved in the pathophysiology of neovascularization (NV) of the eye. Many fields of interest seem to be promising: among them, research regarding new molecular targets involved in the neoangiogenic pathway, together with VEGF-A, would appear to have the potential to bring significant therapeutic improvements in the near future.

\section{Neoangiogenesis in age-related macular degeneration}

Neoangiogenesis is a physiological mechanism involved in many embryonic and adult life processes, such as wound healing, blood vessels reforming during menstruation, placenta formation, etc. Neoangiogenesis may also occur as an unfavourable phenomenon, as is the case, for example, with tumor growth, or in physiologically avascular tissues, such as the cornea, vitreous body or the macular region of the retina. Two major types of ocular NV affect the retina: retinal $\mathrm{NV}$, which is the pathogenetic basis for proliferative diabetic retinopathy, retinal vein occlusions, and retinopathy of prematurity; and subretinal or CNV. ${ }^{5}$ Subretinal and CNV occur in diseases of the outer retina and Bruch's membrane, the most prevalent being nAMD. There is a considerable overlap in the vasoactive molecules involved in retinal and $\mathrm{CNV}$, as both result from a functional dominance of angiogenic factors over antiangiogenic/angiostatic factors, regardless of whether the primary event is an
Correspondence: Federico Galvagni, Department of Biotechnology, Chemistry and Pharmacy, University of Siena, Via Aldo Moro, 2, Siena, Italy.

Phone: +39 0577234961 - Fax: +390577 234254.

E-mail: federico.galvagni@unisi.it

Key words: Choroidal neovascularization; Age-related macular degeneration; New molecular targets.

Contributions: GMT, MB, MO, and FG, selected studies and collected data from the literature, prepared the manuscript and critically revised and approved it.

Conflict of interest: the authors declare no potential conflict of interest.

Received for publication: 26 May 2017

Revision received: 31 July 2017.

Accepted for publication: 3 November 2017

This work is licensed under a Creative Commons Attribution 4.0 License (by-nc 4.0).

(C) Copyright G.M. Tosi et al., 2017

Licensee PAGEPress, Italy

Translational Medicine Reports 2017; 1:6819 doi:10.4081/tmr.6819

increase in angiogenic activity or a decrease in antiangiogenic activity. Although it has not been clearly demonstrated, hypoxia is probably the common primum movens: it provokes the expression of hypoxiainducible factor- $1 \alpha$ (HIF-1 $\alpha)$, which, after binding with HIF-1 $\beta$, becomes an active molecule (HIF-1) capable of promoting the expression of several genes $^{6}$ including VEGF-A, platelet-derived growth factor (PDGF), stromal cell-derived growth factor-1 (SDF-1), and placental growth factor (PIGF).

\section{Challenges to current therapeu- tic strategies}

Despite the success of anti-VEGF-A drugs, modifications and improvements in the management of nAMD are still warranted in relation to several aspects.

\section{Treatment regimens and follow- up frequency}

The treatment of nAMD requires repeated injections and long-term, frequent follow-up. Different treatment schedules have been designed in order to reduce the treatment burden. The most commonly used 
ones are the pro-re-nata (PRN) and the treat-and-extend regimens. ${ }^{7-8}$

Analysis of the CATT and the IVAN trials shows that monthly administration has a better effect on vision than an as needed approach, ${ }^{9-10}$ although clinicians have not yet reached a consensus regarding the best treatment regimen. Nevertheless, there is evidence that shorter injection intervals lead to better visual outcome and steady anatomic improvements. The high costs of frequent anti-VEGF-A drug injections and of a strict regimen of follow-up visits render the treatment of nAMD financially burdensome.

\section{Complications}

Although it is widespread as a technique, the practice of intravitreal injection is not completely free of complications. The intravitreal injection of anti-VEGF-A drugs is related to many adverse events, such as subconjunctival hemorrhages, retinal tears and retinal detachments, vitreous hemorrhages, retinal vascular occlusion, increase of intraocular pressure, cataract, tachyphylaxis, intraocular inflammation and endophthalmitis. ${ }^{11-14}$

Blockage of the VEGF-A pathway itself seems to have potentially negative effects on retinal trophism, due to the role that VEGF-A may play in the maintenance of the choriocapillaris, Müller cells and photoreceptors. ${ }^{15}$ Long-term intravitreal injection of anti-VEGF-A drugs has been correlated with the possible progression of areas of geographic atrophy in patients with nAMD. ${ }^{16}$

\section{Visual loss despite anti-vascular endothelial growth factor A ther- apy}

Inadequate response to anti-VEGF-A drugs is, unfortunately, a common finding and much $\mathrm{CNV}$ remains active despite a protocol of frequent and timely injections. The SEVEN-UP study showed that, approximately 7 years after ranibizumab therapy in the ANCHOR and MARINA trials, one third of patients demonstrated good visual outcomes, whereas another third had poor visual outcomes. ${ }^{17}$ The causes for resistance to anti-VEGF-A therapy are hypothesized to be various: mechanisms of tolerance, tachyphylaxis, compensation by other angiogenic factors, changes in vascular architecture, sustained activation of the complement system, as well as inflammatory response, misdiagnosis and genetic variants. ${ }^{18}$

\section{Future directions: a focus on new molecular targets}

Considering the unresolved problems connected to the current management of patients affected by nAMD, many efforts are being made worldwide in order to design new therapeutic approaches to this multifactorial pathology. It is not the aim of this contribution to discuss new drug delivery methods, which nonetheless represent a very promising field of interest and a valuable potential source of improvement for the current therapeutic strategies. The purpose of this review is to briefly review the promising new molecular targets for neoangiogenesis (Figure 1), inflammation or fibrosis inhibition (Figure 2), that could represent a potential resource in the treatment of nAMD and the related drugs in various stages of development (Table 1).

\section{Other antagonists to the vascular endothelial growth factor A pathway}

\section{Designed ankyrin repeat protein family (DARPin). Designed ankyrin repeat proteins are small, single-domain pro- teins that can selectively bind to a target protein with a higher level of affinity and specificity than an antibody.}

Abicipar pegol (Allergan), previously known as MP0112, is a recombinant protein of the designed ankyrin repeat protein family. It binds to all isoforms of VEGF-A in a similar manner to the currently used anti-VEGF-A agents, but with a higher affinity. A phase II randomized double-blind clinical trial (ClinicalTrials.gov identifier: NCT02181517) evaluated abicipar pegol for AMD compared to ranibizum$a b$. The first results from the phase II study have already been published ${ }^{19}$ : patients received abicipar pegol every month for 3 months, while the control group received ranibizumab monthly for the entire duration of the study. The study was not powered enough to show statistically significant differences between the two drugs, but it showed good results in terms of the efficacy and durability of abicipar pegol. A phase III study evaluating the safety and efficacy of abicipar pegol in patients with nAMD is ongoing (ClinicalTrials.gov identifier: NCT02462486).

TH258. RTH258 (formerly ESBA1008) is a humanized single chain antibody fragment that inhibits all isoforms of VEGF-A. It is a smaller anti-VEGF-A agent than ranibizumab or aflibercept and, because of its high stability and solubility, it is possible to concentrate $\mathrm{RTH} 258$ up to $120 \mathrm{mg} / \mathrm{mL}$, allowing the

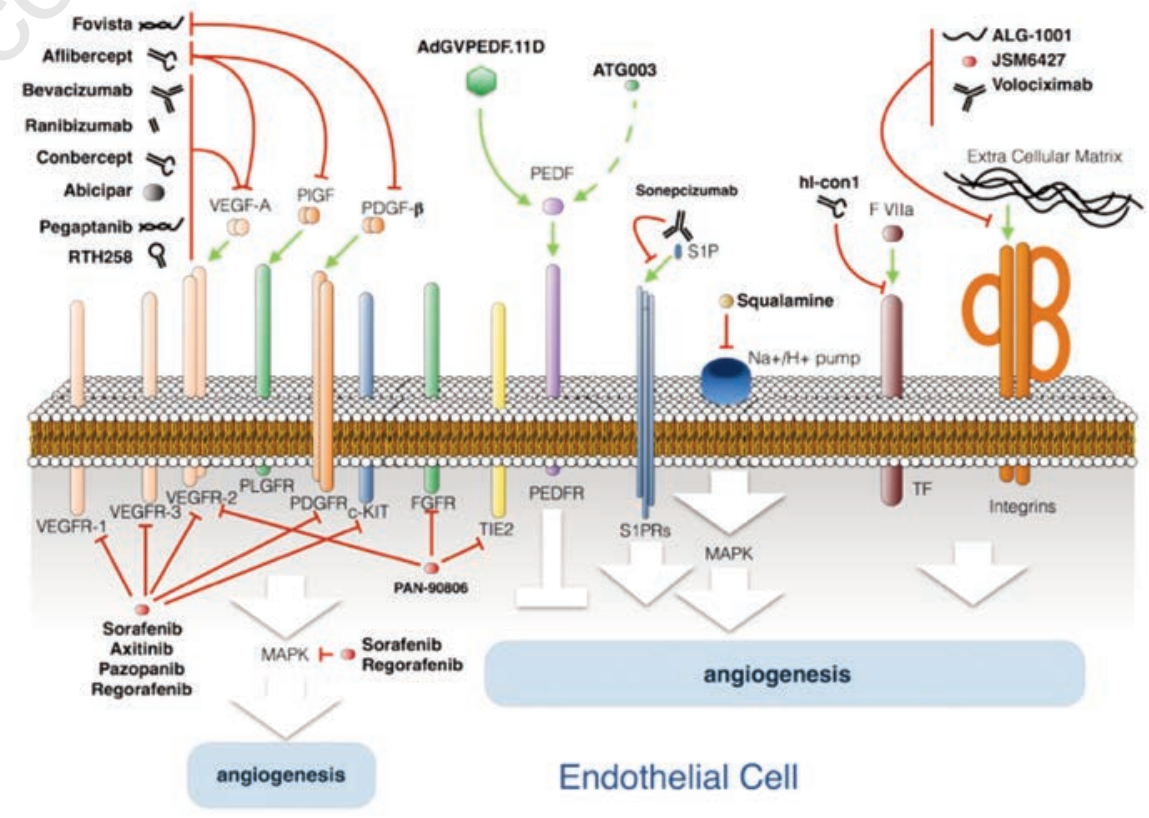

Figure 1. Schematic representation summarizing the antiangiogenic therapy drugs and their targets. See text for details. 
administration of $6 \mathrm{mg}$ in a single 50 $\mu \mathrm{L}$ intravitreal injection. This enables the delivery of a much higher molar dose in the same volume as the current VEGF-A inhibitors in clinical use, potentially supporting the early initiation and prolonged duration of the effects of treatment. Animal studies have shown that the small size of RTH258 leads to faster systemic clearance and lower systemic exposure compared to anti-VEGF-A agents such as ranibizumab and bevacizumab. The smaller size may also allow for better ocular tissue penetration. ${ }^{20-21} \mathrm{~A}$ sixmonth phase I/II prospective multicenter double-masked and randomized clinical trial demonstrated non-inferiority in the change in central subfield thickness at 1 month for the 4.5- and 6.0-mg RTH258 doses compared to ranibizumab, and an increase of 30 days in the median time to retreatment for the 6.0-mg dose. ${ }^{22}$ A phase III clinical trial comparing the efficacy and safety of RTH258 versus aflibercept in subjects with nAMD is ongoing (ClinicalTrials.gov identifier: NCT02307682).

Sphingosine-1-phosphate (S1P) antibody. S1P is a bioactive lipid mediator whose biological activities are moderated by the surface receptors found on endothelial cells. Retinal pigment epithelium (RPE) may release and store S1P in the retina and could contribute to the pathological angiogenesis, vascular permeability, fibrosis and inflammatory responses associated with nAMD. ${ }^{23}$ Sonepcizumab, a monoclonal antibody that selectively binds to S1P, was evaluated in animal models with oxygeninduced ischemic retinopathy and was found to cause a decreased inflow of macrophages into the retina, the suppression of retinal $\mathrm{NV}$, and reduced CNV after laser disruption of Bruch's membrane. ${ }^{23}$ iSONEP (LPath, Inc, San Diego, CA, USA), the ocular formulation of sonepcizumab, was evaluated in a phase II clinical trial which did not meet its primary or key secondary endpoints. nAMD patients who had not responded adequately to existing antiVEGF-A therapies, including Lucentis, Avastin and Eylea, did not show any statistically significant improvement in visual acuity when treated with iSONEP as an adjunctive or sole therapy (ClinicalTrials.gov identifier: NCT01414153).

Squalamine lactate. Squalamine is a small molecule derived from the cartilage of the dogfish shark (Squalus acan- thias), which acts against the development of aberrant NV, by inhibiting downstream signaling pathways of multiple growth factors, including VEGFA, PDGF and basic fibroblast growth factor (bFGF). A phase III clinical trial evaluating the efficacy and safety of ophthalmic squalamine lactate solution $0.2 \%$ (OHR-102, Ohr Pharmaceuticals, New York, NY), administered twice daily in association with intravitreal ranibizumab, is currently recruiting patients (ClinicalTrials.gov identifier: NCT02727881).

Small interfering RNA (siRNA). With the aim of down-regulating the production of VEGF-A and the activity of VEGF receptors (VEGFRs), siRNA technology has been tested for the treatment of AMD. siRNA are short RNA molecules that, once incorporated into the RNA-induced silencing complex, are able to guide the degradation of specific mRNAs. Naked siRNAs targeting VEGF-A (bevasiranib, Opko Health Inc., Miami, FL, USA) or one of its receptors, VEGFR1 (AGN 211745, previously siRNA-027; Allergan Inc., Irvine, CA, USA), were administered intravitreously and tested as drug candidates in clinical trials for $\mathrm{CNV}$ due to
AMD. The preliminary safety data of these two compounds were encouraging, but they failed to meet key efficacy parameters in further studies. ${ }^{24}$

\section{Platelet-derived growth factor inhibition}

In vitro studies have demonstrated the role of PDGF in the process of angiogenesis. ${ }^{25}$ It has been shown to promote the migration and proliferation of endothelial cells, as well as an increase in the recruitment of pericytes, which have been demonstrated to play a central role in protecting the endothelial cells against VEGF-A inhibition. ${ }^{26}$

The involvement of PDGF in the ocular $\mathrm{NV}$ process has been demonstrated ${ }^{27}$ and seems to be related to the entry of platelets and monocytes into the vitreous and subretinal space upon injury to the blood-retina barrier, with subsequent platelet aggregation and PDGF discharge. Interleukins such as IL-1 and TGF- $\beta$ released from activated macrophages may cause further synthesis and release of PDGF.

- Fovista. Ophthotech (New York, U.S.A.) has developed a high affinity
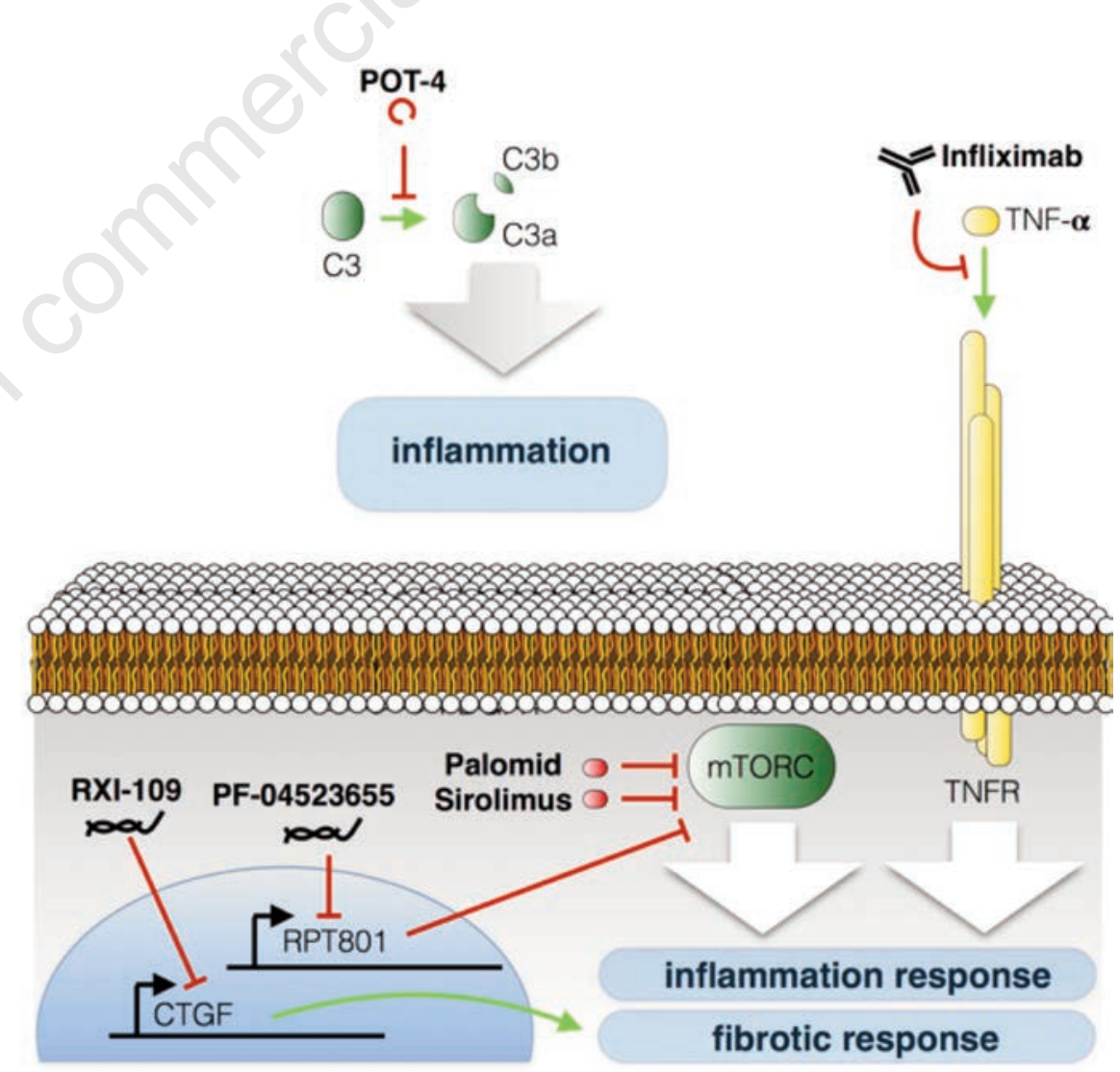

Figure 2. Schematic representation summarizing the anti-inflammatory and anti-fibrotic therapy drugs and their targets. See text for details. 
PDGF antagonist called E10030 (Fovista). This molecule binds to PDGF and prevents it from binding to its receptor PDGFR- $\beta$. In vitro/in vivo studies using this compound have shown its effectiveness, in combination with an anti-VEGF-A agent, in preventing the formation of CNV, as well as in stripping pericytes from vessels that have already formed, leading to their regression. Phase-I and phase-II clinical trials investigating the safety and tolerability, and safety and efficacy, of intravitreal injection of Fovista in humans, respectively in combination with anti-VEGF-A agents or as a monotherapy, have been completed, showing Fovista as a possible future therapeutic agent when used in combination with anti-VEGF-A drugs (Clinical Trials.gov identifier: NCT00569140) (ClinicalTrials.gov identifier: NCT01089517). Two phaseIII, randomized, double-blind clinical trials evaluating the safety and efficacy of the intravitreous administration of Fovista in combination with ranibizum$\mathrm{ab}$, compared to ranibizumab monotherapy, have recently been terminated (ClinicalTrials.gov identifier: NCT01944839) (ClinicalTrials.gov identifier: NCT01940900). Unfortunately, Ophthotech Corporation announced that the addition of Fovista to a monthly Lucentis regimen did not result in benefit as measured by the mean change in visual acuity at the 12 month time point.

\section{Tyrosine kinase inhibition}

Since, as suggested by recent research, the activity of VEGF-A and other angiogenic factors is regulated by tyrosine kinases, kinase inhibitors may be used to target VEGFRs. The high homology between VEGFRs and PDGFRs allows many kinase inhibitors to simultaneously block both. ${ }^{28}$

- Axitinib. Axitinib is a multi-receptor tyrosine kinase inhibitor that has marked inhibitory effects on VEGFR2, PDGFR- $\beta$ and c-KIT receptors. Axitinib is currently approved for use in advanced human renal cell carcinoma. Because of its anti-VEGFR and antiPDGFR- $\beta$ action, its use in nAMD treatment has been suggested. It recently showed good efficacy at a low dose in a laser-induced CNV model in rats. ${ }^{29}$ - Pazopanib. Pazopanib (GlaxoSmithKline, Brentford, UK) (GW786034) is a small molecule that has been investigated as an eye drop in $\mathrm{CNV}$, due to its ability to act as an inhibitor of VEGFRs 1, 2, and 3; PDGF- $\alpha$ and $\beta$; and the stem cell factor receptor c-KIT. ${ }^{30-34}$ A multi-country, randomized, double-masked, dose-ranging study phase IIb clinical trial was completed in 2015. It demonstrated the inferiority of daily pazopanib eye drops compared to intravitreal injection of ranibizumab in the treatment of nAMD. ${ }^{35}$

Regorafenib. Regorafenib (Bayer Healthcare, Leverkusen, Germany) is an eye drop multikinase inhibitor (MKI) which targets several angiogenic kinases, including VEGFR-2, VEGFR-1, VEGFR-3, fibroblast growth factor receptor 1 (FGFR1), PDGFR- $\beta$ and the mutant oncogenic kinases c-KIT, RET and B-RAF. It showed positive results in a phase I clinical trial in healthy volunteers and a phase II clinical trial is currently in progress. ${ }^{36}$

Pan 90806. Pan 90806 (PanOptica) is a MKI of VEGFR-2, FGFR1-3, TIE2, and of the autophosphorylation of other pro-angiogenic tyrosine kinase receptors. This molecule was initially investigated as an oral antineoplastic agent but showed poor results. Subsequently it was adopted as an eye drop for AMD and diabetic retinopathy, since it is a small molecule with high permeability and has the ability to concentrate high doses in the active form at the posterior pole. Phase I/II studies are currently ongoing (ClinicalTrials.gov identifier: NCT02022540).

Sorafenib. Sorafenib is an oral MKI whose antiangiogenic action inhibits VEGFR-1, -2, and -3, as well as PDGFR- $\beta$, RAF kinase and c-KIT. ${ }^{37}$ In a study using a mouse model of CNV,

Table 1. Approved and not-yet-approved drugs for age-related macular degeneration treatment.

\begin{tabular}{|c|c|c|c|}
\hline Drug & Type of molecule & Target & Clinical trial \\
\hline Drug & Type of molecule & Target & Clinical trial \\
\hline Bevacizumab & Monoclonal antibody & VEGF-A & Approved \\
\hline Aflibercept & Chimeric soluble receptor & VEGF-A/PIGF & Approved \\
\hline Conbercept & Chimeric soluble receptor & VEGF-A & Approved \\
\hline Ranibizumab & Fab fragment of antibody & VEGF-A & Approved \\
\hline Pegaptanib & Pegylated aptaner & VEGF-A & Approved \\
\hline Abicipar & Single-domain protein & VEGF-A & Phase III \\
\hline RTH258 & Single-Chain Antibody Fragment & VEGF-A (all isoforms) & Phase III \\
\hline Axitinib & Small molecule & VEGFR-2/PDGFR-ß/c-KIT & Phasel/II \\
\hline Pazopanib & Small molecule & VEGFR-1,2,3/PDGFR $\alpha, \beta, c-K I T$ & Phase II \\
\hline Regorafenib & Small molecule & VEGFR-1,2,3/c-KIT/PDGFRß/FGFR1/MAPK & K Phase II \\
\hline Pan 90806 & Small molecule & VEGFR-1,2,3/TIE2/FGFR1,2,3 & Phase I/II \\
\hline Sorafenib & Small molecule & VEGFR-1,2,3/c-KIT/PDGFRß/RAF & Phase II \\
\hline Sonepcizumab & Monoclonal antibody & S1P & Phase II \\
\hline Squalamine & Small molecule & proton pumps & Phase III \\
\hline Fovista & Pegylated Aptamer & PDGF & Phase III \\
\hline AdGVPEDF.11D & adenoviral vector & PEDF expression & Phase I \\
\hline ATG003 & Small molecule & nAchR/PEDF expression & Phase II \\
\hline JSM6427 & Small molecule & $\alpha v \beta 1$ integrins & Phase I \\
\hline Volociximab & Chimeric monoclonal antibody & $\alpha 5 \beta 1$ integrin & Phase I \\
\hline ALG-1001 & Oligopeptide & $\alpha 5 \beta 1, \alpha v \beta 3$ and $\alpha v \beta 5$ integrins & Phase I \\
\hline Infliximab & Monoclonal antibody & TNF- $\alpha$ & Phase II \\
\hline POT-4 & Oligopeptide & Complement C3 & Phase I \\
\hline Sirolimus & Small molecule & mTOR & Phase II \\
\hline Palomid 529 & Small molecule & mTORCl & Phase I \\
\hline PF-04523655 & siRNA & RTP801 expression & Phase II \\
\hline RXI-109 & sd-rxRNA & CTGF expression & Phase I \\
\hline hI-conl & Chimeric protein & Tissue factor & Phase II \\
\hline
\end{tabular}

VEGF-A, vascular endothelial growth factor A; PIGF, placental growth factor; VEGFR, vascular endothelial growth factor receptor; PDGF, platele derived growth factor; PDGFR, platelet derived growth factor receptor; FGFR, fibroblast growth factor receptor; KIT, v-kit feline sarcoma viral oncogene homolog; RAF, v-raf-1 murine leukemia viral oncogene homolog 1; TIE, TEK tyrosine kinase endothelial; MAPK, mitogen-activated protein kinase; PEDF, Pigment epithelium-derived factor; mTOR, mammalian target of rapamycin; mTORC, mTOR complex; TNF- $\alpha$, Tumor necrosis factor; S1P, Sphingosine-1-phosphate; nAChR, Nicotinic acetylcholine receptor; C3, Component 3. 
sorafenib showed the ability to decrease the extent of CNV in a dose-dependent manner. ${ }^{38}$ In two case reports, sorafenib was administered orally in combination with intravitreal anti-VEGF-A treatment and all patients appeared to gain some benefit. ${ }^{39-40}$

\section{Pigment epithelium-derived factor}

PEDF is a cell survival factor secreted by the RPE, widely expressed in the central and peripheral nervous system, ${ }^{41,42}$ and apparently an endogenous inhibitor of angiogenesis in the eye. ${ }^{43} \mathrm{~A}$ critical balance appears to exist between PEDF and VEGFA, with PEDF counteracting the angiogenic potential of VEGF-A. A physiological decrease in PEDF occurring in the elderly may disrupt this balance and create a permissive environment for the formation of $\mathrm{CNV}$ in AMD. ${ }^{44}$ PEDF gene polymorphisms may also contribute to AMD. ${ }^{45}$

$A T G 003$. PEDF protein expression has been found to be decreased in RPE from smoker patients with AMD compared to controls. ${ }^{46}$ It has also been reported that nicotine, a potent angiogenic agent, increased the VEGF-A/PEDF ratio in the RPE through nicotinic acetylcholine receptor (nAchR). ${ }^{47}$ ATG003 (CoMentis, formerly Athenagen, South San Francisco, CA, USA) is a topical mecamylamine that antagonizes the nAchR pathway which mediates angiogenesis. It was designed as an eye drop therapy for AMD, and phase II clinical trials testing its safety and efficacy were completed in 2010 (ClinicalTrials.gov identifier:

NCT00414206)

(Clinicaltrials.gov identifier: NCT00607750), although no results have been published yet.

- AdGVPEDF.11D. An adenoviral vector containing complementary DNA encoding human PEDF, known as AdGVPEDF.11D (GenVec, Inc., Gaithersburg, MD, USA), seems to allow the expression of large amounts of PEDF in the target tissue and inhibit ocular $\mathrm{NV}$ in murine AMD models. ${ }^{48}$ Intravitreal injection of AdGVPEDF.11D results in the local production of PEDF. A phase I single-dose trial enrolled 28 patients with severe nAMD. ${ }^{49}$ This clinical study suggested that antiangiogenic activity may last for up to 6 months after a single intravitreous injection, since half of the lesions treated did not change in size from baseline. However, no further studies have corroborated this hypothesis.

\section{Integrin receptor antagonists}

Integrins are transmembrane proteins that mediate the attachment between cells and the surrounding extracellular matrix. Their ligands are fibronectin, vitronectin, collagen and laminin. ${ }^{50}$ Integrins localized at the apical surface of RPE bind to ligands in the interphotoreceptor matrix ${ }^{51}$ and participate in the interactions between photoreceptors and the RPE. ${ }^{52}$ During aging, the dissociation between the interphotoreceptor matrix and retinal cells seems to contribute to decreasing the supply of oxygen, nutrients and growth factors from the choroidal or retinal vessels, as well as to inhibit the phagocytosis of photoreceptor outer segments. ${ }^{53}$ It has been shown that $\alpha 5 \beta 1, \alpha v \beta 3$ and $\alpha v \beta 5$ integrins are expressed in $\mathrm{CNV}$ tissue, ${ }^{54}$ and many studies have demonstrated a possible role for their antagonists in preventing ocular NV. ${ }^{55-59}$

- SM6427. JSM6427 is a small molecule antagonist of the $\alpha 5 \beta 1$ integrin, which has been shown to inhibit retinal and $\mathrm{CNV}$ in preclinical models. ${ }^{60} \mathrm{~A}$ phase I clinical trial of JSM6427 for nAMD has been completed (ClinicalTrials.gov identifier: NCT00536016). Although encouraging results were obtained, no further studies have evaluated JSM6427.

- Volociximab. Volociximab (Ophthotech Corporation, Princeton, NY, USA) is a chimeric monoclonal antibody specifically blocking the binding of fibronectin to the $\alpha 5 \beta 1$ integrin. A phase I study evaluating the safety of intravitreal volociximab combined with ranibizumab for nAMD (ClinicalTrials.gov identifier: NCT00782093) revealed good improvements in visual acuity at nine weeks, although the results did not distinguish the independent contributions of ranibizumab and volociximab.

- ALG-1001. ALG-1001, also known as Luminate (Allegro Ophthalmics, San Juan Capistrano, CA, USA), is a synthetic oligopeptide that targets $\alpha 5 \beta 1$, $\alpha v \beta 3$ and $\alpha v \beta 5$ integrins. It has been investigated for 3 indications: nAMD, diabetic macular edema and vitreomacular traction. In a phase Ib clinical trial ALG-1001 used in combination with ranibizumab for the treatment of nAMD the drug was found to be safe. ${ }^{61}$

\section{Anti-inflammatory and anti- immune therapies}

Over the last few years many authors have identified inflammatory mechanisms and immune responses as central features in all AMD phenotypes, consolidating the hypothesis that AMD is a singular disease with multiple outcomes. ${ }^{62-64}$

Investigating the role of immune response in the pathogenesis of ocular NV may therefore represent a promising field in the search for new targets for AMD treatments (Figure 2).

Infliximab. Tumor necrosis factor alpha (TNF- $\alpha$ ) plays a central role in inflammation, apoptosis and immune reactions. The anti-TNF- $\alpha$ monoclonal antibody infliximab (Remicade, Centocor, Inc., Horsham, PA, USA) is commonly used to treat various inflammatory diseases. Intravitreal infliximab has been reported to inhibit laser-induced CNV in rats and has been safely administered up to a dose of $2 \mathrm{mg}$ in the rabbit eye. ${ }^{65-66}$ Intravitreal infliximab was also administered to three patients with nAMD, resulting in improved visual acuity and central foveal thickness on Optical Coherence Tomography (OCT). ${ }^{67}$

POT-4. The alternative complement pathway has also been implicated in the development of AMD. ${ }^{68}$ POT-4 is a potent inhibitor of complement factor C3 activation. A phase I clinical trial was designed to evaluate the safety and tolerability of POT-4 administered via intravitreal injections (ClinicalTrials.gov identifier: NCT00473928). Preliminary results indicated that intravitreal POT-4 is safe and well-tolerated and supported its administration in larger randomized clinical trials in order to further define its efficacy profile. Even though no significant improvement in visual acuity was observed, there was no significant visual loss either. ${ }^{69}$

Sirolimus. Mammalian target of rapamycin (mTOR) is an evolutionarily conserved serine/threonine kinase which appears to function as a central node in a signaling cascade directing the integration of diverse environmental inputs into the immune microenvironment. ${ }^{70}$ Sirolimus (previously known as rapamycin, Santen Pharmaceutical, Inc., Osaka, Kapan, and MacuSight, Inc., Union City, CA) was discovered in the 1970 s as a soil bacterium metabolite. It was collected on Easter Island (Rapa Nui) and was originally developed as a macrolide antifungal agent. Subsequently, it was found to possess potent immunosuppressive and antiproliferative properties due to its ability to bind to the protein FKBP12. The resulting sirolimus-FKBP12 complex binds 
to and inhibits mTOR. ${ }^{71}$ It is used as an anticancer drug against several solid tumors and an immunosuppressive agent in the transplantation of various organs, especially kidneys. ${ }^{72}$ Preclinical studies in experimental models have shown promising results regarding the use of this pharmacological agent to inhibit ocular neoangiogenesis. ${ }^{73-74}$ Early phase I/II studies have subsequently provided encouraging safety and efficacy data (ClinicalTrials.gov identifier: NCT00712491). A phase II study (EMERALD) of an ocular sirolimus (rapamycin) formulation in combination with ranibizumab in patients with AMD has been terminated: the last update was in June 2013 (ClinicalTrials.gov identifier: NCT00766337).

- Palomid 529. Unlike Sirolimus, which binds to mTOR Complex 1 (mTORC1), Palomid 529 is capable of blocking both mTORC1 and mTORC2. A phase I clinical trial enrolled five participants with nAMD who were refractory to intravitreal anti-VEGF-A, and received three consecutive monthly subconjunctival doses of $1.9 \mathrm{mg}$ Palomid 529. Unfortunately, compared to baseline no clinically important changes were observed in best-corrected visual acuity, fluorescein leakage pattern, $\mathrm{CNV}$ size at indocyanine green angiography, or autofluorescence patterns at fundus autofluorescence. ${ }^{75}$

- PF-04523655. PF-04523655 is a 19ribonucleotide double-stranded siRNA which inhibits the expression of the hypoxia-inducible gene, RTP801, an inhibitor mTOR signaling. ${ }^{76}$ A single intrevitreal injection of PF-0523655 $\leq 3000 \mathrm{mg}$ seemed to be safe and welltolerated in a phase I study involving 27 patients with AMD which had been unresponsive to prior treatment. ${ }^{77} \mathrm{~A}$ phase II clinical trial, the multicenter and randomized MONET study, sought to prove the efficacy of PF-04523655 in combination with Lucentis. It demonstrated the superiority of PF04523655+ranibizumab over ranibizumab monotherapy in terms of best corrected visual acuity improvement. $^{78}$

\section{Connective tissue growth factor inhibition}

The 38-KDa cysteine-rich polypeptide connective tissue growth factor (CTGF), which has a pleiotropic and cell type-specif- ic role, was first isolated in human umbilical vein endothelial cells. ${ }^{79} \mathrm{CTGF}$ is considered to be involved in the pathological synthesis of peri-retinal fibrous tissue in AMD patients. $^{80}$

RXI-109. RXI-109 (RXi Pharmaceuticals, Marlborough, MA) is a self-delivering RNAi compound (sd-rxRNA), designed to reduce the expression of CTGF and developed to reduce dermal scarring following planned surgery. A study designed to evaluate the safety, tolerability and clinical activity of RXI-109 administered by intravitreal injection, in order to reduce the progression of subretinal fibrosis in subjects with advanced nAMD degeneration, is currently recruiting patients (ClinicalTrials.gov identifier: NCT02599064).

\section{Tissue factor inhibition}

Tissue factor (TF) is expressed in neovascular endothelium but not in the normal vasculature of mouse and pig models. - hI-con1. Factor VII-verteporfin has been tested with the aim of improving the therapeutic results of photodynamic therapy in rats, due to its ability to bind tightly and specifically to TF, which is expressed in the endothelial cells of CNV but not in the normal vasculature. ${ }^{81} \mathrm{hI}$-con1 (Iconic Therapeutics Inc. South San Francisco, CA, USA), a synthetic molecule composed of factor VII conjugated to an antibody $\mathrm{Fc}$, binds to $\mathrm{TF}$ and selectively destroys pathologic blood vessels. A phase II, randomized, double-masked, multi-center study evaluating the administration of repeated intravitreal injections of hI-con1 in patients with CNV secondary to AMD has recently been completed and the results are pending (ClinicalTrials.gov identifier: NCT02358889).

\section{CD93}

Human CD93 is a 652 amino acid surface glycoprotein with a predicted molecular mass of $68 \mathrm{kDa}$; it is rich in proline and charged amino acids. ${ }^{82-83}$ CD93 expression has been found in several cell types, including the vascular endothelium. ${ }^{84-85}$ Moreover, the soluble EGF-like domain of CD93, which is the product of ectodomain cleavage or shedding, has recently been discovered to be an angiogenic factor. ${ }^{86}$ The monoclonal antibody 4E1, binding to CD93, is capable to selectively inhibit blood vessel formation both in vitro and in vivo, without affecting endothelial cell survival. ${ }^{87}$

CD93 expression has recently been observed in the endothelial cells of AMDrelated $\mathrm{CNV}$ and the soluble CD93 domain has been shown to be overexpressed in the aqueous humor of AMD patients, suggesting that CD93 may represent a potential new antiangiogenic target in the treatment of $\mathrm{CNV}^{88}$

\section{HTRA1}

HTRA1 is a serine protease involved in protein quality control and cell fate. HTRA1 expression has been found to be significantly higher in CNV patients compared to controls. After treatment with antiVEGF-A, it returned to control levels. ${ }^{89}$ The ability of HTRA1 to regulate extracellular matrix proteoglycan degradation and TGF$\beta$ activity appears to allow it to modulate AMD development. ${ }^{90-91}$ HTRA1 can be considered an innovative target. This hypothesis is supported by the discovery of the antibody 94, which is an inhibitor of this protein. $^{92}$

\section{Conclusions}

Over the last decade, the management of AMD has changed significantly due to the advent of anti-VEGF-A therapy. However, treatment with anti-VEGF-A agents rarely brings about an improvement in visual acuity. The study of the pathogenetic mechanisms of this disease has led to the discovery of several proteins and factors, which could be used as new molecular targets in AMD therapy.

A great effort has been made to inhibit neoangiogensis with several drugs now in advanced clinical trials (Figure 1) targeting new antigens with complementary functions, which could offer novel opportunities to circumvent resistance mechanisms and improve the anti-VEGF-A therapy.

The unsuccessful outcomes of the antiVEGF-A therapy have often been attributed to development of subretinal fibrosis and inflammatory response. Other interesting drugs seem to come from these fields of research (Figure 2), with at least four compounds in clinical phase II (Infliximab, Sirolimus, PF-04523655 and hI-con1).

Future directions in AMD treatment will have to focus now on applying combinations of drugs with different angiogenic and non-angiogenic targets, with the aim of achieving disease stabilization or regression, improving visual acuity, and shortening injection intervals. 


\section{References}

1. Wong WL, Su X, Li X, et al. Global prevalence of age-related macular degeneration and disease burden projection for 2020 and 2040: a systematic review and meta-analysis. Lancet Glob Health 2014;2:106-16.

2. Kwak N, Okamoto N, Wood JM, Campochiaro PA. VEGF is an important stimulator in a model of choroidal neovascularization. Invest Ophthalmol Vis Sci 2000;41:3158-64.

3. Krzystolik MG, Afshari MA, Adamis AP, et al. Prevention of experimental choroidal neovascularization with intravitreal anti-vascular endothelial growth factor antibody fragment. Arch Ophthalmol 2002;120:338-46.

4. Rosenfeld PJ, Brown DM, Heier JS, et al. Ranibizumab for neovascular agerelated macular degeneration. New Engl J Med 2006;355:1419-31.

5. Campochiaro PA. Molecular pathogenesis of retinal and choroidal vascular diseases. Prog Retin Eye Res 2015;49:67-81.

6. Stone J, Itin A, Alon $\mathrm{T}$, et al. Development of retinal vasculature is mediated by hypoxia-induced vascular endothelial growth factor (VEGF) expression by neuroglia. J Neurosci 1995;15:4738-47.

7. Lalwani GA, Rosenfeld PJ, Fung AE, et al. A variable-dosing regimen with intravitreal ranibizumab for neovascular age-related macular degeneration: year 2 of the PrONTO Study. Am J Ophthalmol 2009;148:43-58.

8. Fung AT, Kumar N, Vance SK, et al. Pilot study to evaluate the role of highdose ranibizumab $2.0 \mathrm{mg}$ in the management of neovascular age-related macular degeneration in patients with persistent/recurrent macular fluid $<30$ days following treatment with intravitreal anti-VEGF therapy (the LAST Study). Eye (Lond) 2012;26:1181-87.

9. Chakravarthy U, Harding SP, Rogers $\mathrm{CA}$, et al. IVAN study investigators. Alternative treatments to inhibit VEGF in age-related choroidal neovascularisation: 2-year findings of the IVAN randomised controlled trial. Lancet 2013;382:1258-67.

10. Martin DF, Maguire MG, Fine SL, et al. Ranibizumab and bevacizumab for treatment of neovascular age-related macular degeneration: two-year results. Ophthalmology 2012;7:1388-98.

11. Day S, Acquah K, Mruthyunjaya P, et al. Ocular complications after anti-vascular endothelial growth factor therapy in Medicare patients with age-related macular degeneration. Am J Ophthalmol 2011;152:266-72.

12. Sharma S, Johnson D, Abouammoh M, et al. Rate of serious adverse effects in a series of bevacizumab and ranibizumab injections. Can J Ophthalmol 2012;47: 275-9.

13. Pershing S, Bakri SJ, Moshfeghi DM. Ocular hypertension and intraocular pressure asymmetry after intravitreal injection of anti-vascular endothelial growth factor agents. OSLI Retina 2013;44:460-4.

14. Gregori NZ, Flynn HW Jr, Schwartz $\mathrm{SG}$, et al. Current infectious endophthalmitis rates after intravitreal injections of anti-vascular endothelial growth factor agents and outcomes of treatment. OSLI Retina 2015;46:643-8.

15. Saint-Geniez M, Kurihara T, Sekiyama $E$, et al. An essential role for RPEderived soluble VEGF in the maintenance of the choriocapillaris. P Natl Acad Sci USA 2009;106:18751-6.

16. Gemenetzi M, Lotery AJ, Patel PJ. Risk of geographic atrophy in age-related macular degeneration patients treated with intravitreal anti-VEGF agents. Eye (Lond) 2017;31:1-9.

17. Rofagha S, Bhisitkul RB, Boyer DS, et al. SEVEN-UP Study Group. Sevenyear outcomes in ranibizumab-treated patients in ANCHOR, MARINA, and HORIZON: a multicenter cohort study (SEVEN-UP). 2013;120:2292-99.

Ophthalmology

18. Yang S, Zhao J, Sun X. Resistance to anti-VEGF therapy in neovascular agerelated macular degeneration: a comprehensive review. Drug Des Dev Ther 2016;10:1857-67.

19. Souied EH, Devin F, Mauget -Faÿsse M, et al. MP0112 Study Group. Treatment of exudative age-related macular degeneration with a designed ankyrin repeat protein that binds vascular endothelial growth factor: a phase I/II study. Am J Ophthalmol 2014;158:724-32.

20. Tietz J, Schmid G, Konrad J, et al. Affinity and potency of RTH258 (ESBA1008), a novel inhibitor of vascular endothelial growth factor a for the treatment of retinal disorders. Invest Ophthalmol Vis Sci 2015;1501.

21. Gaudreault J, Gunde T, Floyd HS, et al. Preclinical pharmacology and safety of ESBA1008, a single-chain antibody fragment, investigated as potential treatment for age related macular degeneration. Invest Ophthalmol Vis Sci 2012;53:3025.

22. Holz FG, Dugel PU, Weissgerber G, et al. Single-chain antibody fragment
VEGF inhibitor RTH258 for neovascular age-related macular degeneration: a randomized controlled study. Ophthalmology 2016;123:1080-9.

23. Sabbadini RA. Sphingosine-1-phosphate antibodies as potential agents in the treatment of cancer and age-related macular degeneration. Brit J Pharmacol 2011;162:1225-38.

24. Guzman-Aranguez A, Loma P, Pintor J. Small-interfering RNAs (siRNAs) as a promising tool for ocular therapy. Brit J Pharmacol 2013;170:730-47.

25. Sato N, Beitz JG, Kato J, et al. Plateletderived growth factor indirectly stimulates angiogenesis in vitro. Am J Pathol 1993;142:1119-30.

26. Hellberg C, Ostman A, Heldin $\mathrm{CH}$. PDGF and vessel maturation. Recent Res Cancer 2010;180:103-14.

27. Robbins SG, Mixon RN, Wilson DJ, et al. Platelet-derived growth factor ligands and receptors immunolocalized in proliferative retinal diseases. Invest Ophthalmol Visual Sci 1994;35:364963.

28. Takahashi K, Saishin Y, Saishin Y, King AG, et al. Suppression and regression of choroidal neovascularization by the multitargeted kinase inhibitor pazopanib. Arch Ophthalmol 2009; 127:494-9.

29. Giddabasappa A, Lalwani K, Norberg $\mathrm{R}$, et al. Axitinib inhibits retinal and choroidal neovascularization in in vitro and in vivo models. Exp Eye Res 2016;145:373-9.

30. McLaughlin MM, Paglione MG, Slakter $\mathrm{J}$, et al. Initial exploration of oral pazopanib in healthy participants and patients with age-related macular degeneration. JAMA Ophthalmol 2013;131:1595-601.

31. Thakur A, Scheinman RI, Rao VR, Kompella UB. Pazopanib, a multitargeted tyrosine kinase inhibitor, reduces diabetic retinal vascular leukostasis and leakage. Microvasc Res 2011;82:34650 .

32. Danis R, McLaughlin MM, Tolentino M, et al. Pazopanib Eye Drops Study Group. Pazopanib eye drops: a randomized trial in neovascular age-related macular degeneration. Brit J Ophthalmol 2014;98:172-8.

33. Kumar R, Knick VB, Rudolph SK, et al. Pharmacokinetic-pharmacodynamic correlation from mouse to human with pazopanib, a multikinase angiogenesis inhibitor with potent antitumor and antiangiogenic activity. Mol Cancer Ther 2007;6:2012-21.

34. Kernt M, Thiele S, Neubauer AS, et al. Inhibitory activity of ranibizumab, 
sorafenib, and pazopanib on lightinduced overexpression of plateletderived growth factor and vascular endothelial growth factor A and the vascular endothelial growth factor A receptors 1 and 2 and neuropilin 1 and 2 . Retina 2012;32:1652-63.

35. Csaky KG, Dugel PU, Pierce AJ, et al. Clinical evaluation of pazopanib eye drops versus ranibizumab intravitreal injections in subjects with neovascular age-related macular degeneration. Ophthalmology 2015;122:579-88.

36. Pecen PE, Kaiser PK. Current phase $1 / 2$ research for neovascular age-related macular degeneration. Curr Opin Ophthalmol 2015;26:188-93.

37. Wilhelm SM, Adnane L, Newell P, et al. Preclinical overview of sorafenib, a multikinase inhibitor that targets both Raf and VEGF and PDGF receptor tyrosine kinase signaling. Mol Cancer Ther 2008; 7:3129-40.

38. Chung EJ, Yoo S, Lim HJ, et al. Inhibition of choroidal neovascularisation in mice by systemic administration of the multikinase inhibitor, sorafenib. Brit J Ophthalmol 2009;93:958-63.

39. Diago T, Pulido JS, Molina JR, et al. Ranibizumab combined with low-dose sorafenib for exudative age-related macular degeneration. Mayo Clin Proc 2008;83:231-4.

40. Kernt M, Staehler M, Stief C, et al. Resolution of macular oedema in occult choroidal neovascularization under oral Sorafenib treatment. Acta Ophthalmol 2008;86:456-8.

41. Mousa SA, Lorelli W, and Campochiaro PA. Role of hypoxia and extracellular matrix-integrin binding in the modulation of angiogenic growth factors secretion by retinal pigmented epithelial cells. J Cell Biochem 1999;74:135-43.

42. Tombran-Tink J, Shivaram SM, Chader GJ, et al. Expression, secretion, and age-related downregulation of pigment epithelium-derived factor, a serpin with neurotrophic activity. J Neurosci 1995; 15:4992-5003.

43. Holekamp NM, Bouck N, Volpert O. Pigment epithelium-derived factor is deficient in the vitreous of patients with choroidal neovascularization due to age-related macular degeneration. Am J Ophthalmol 2002;134:220-7.

44. Steinle JJ, Sharma S, Chin VC. Normal aging involves altered expression of growth factors in the rat choroid. J Gerontol A Biol Sci Med Sci 2008;63:135-40.

45. Lin JM, Wan L, Tsai YY, et al. Pigment epithelium-derived factor gene Met72Thr polymorphism is associated with increased risk of wet age-related macular degeneration. Am J Ophthalm 2008;145:716-21.

46. Christen WG, Glynn RJ, Manson JE, et al. A prospective study of cigarette smoking and risk of age-related macular degeneration in men. JAMA 1996;276:1147-51.

47. Pons $M$ and Marin-Castano ME. Nicotine increases the VEGF/PEDF ratio in retinal pigment epithelium: a possible mechanism for $\mathrm{CNV}$ in passive smokers with AMD. Invest Ophthalmol Vis Sci 2011;52:3842-53.

48. Mori K, Gehlbach P, Ando A, et al. Regression of ocular neovascularization in response to increased expression of pigment epithelium derived factor. Invest Ophthalmol Vis Sci 2002;43:2428-34.

49. Campochiaro PA, Nguyen QD, Shah $\mathrm{SM}$, et al. Adenoviral vector-delivered pigment epithelium-derived factor for neovascular age-related macular degeneration: results of a phase I clinical trial. Hum Gene Ther 2006;17:167-76.

50. Hynes RO. The extracellular matrix: not just pretty fibrils. Science 2009;326: 1216-9.

51. Nandrot EF, Kim Y, Brodie SE, et al. Loss of synchronized retinal phagocytosis and age-related blindness in mice lacking $\alpha v \beta 5$ integrin. J Exp Med 2004;200:1539-45.

52. Mallavarapu $M$ and Finnemann SC. Neural retina and MerTK-independent apical polarity of alphavbeta5 integrin receptors in the retinal pigment epithelium. Adv Exp Med Biol 2010;664:12331.

53. Al-Ubaidi MR, Naash MI, Conley SM. A perspective on the role of the extracellular matrix in progressive retinal degenerative disorders. Invest Ophth Vis Sci 2013;54:8119-24.

54. Friedlander M, Theesfeld CL, Sugita M, et al. Involvement of integrins $\alpha v \beta 3$ and $\alpha v \beta 5$ in ocular neovascular diseases. Proc Natl Acad Sci USA 1996;93:97649.

55. Luna J, Tobe $\mathrm{T}$, Mousa $\mathrm{SA}$, et al. Antagonists of integrin alpha $\mathrm{v}$ beta 3 inhibit retinal neovascularization in a murine model. Lab Invest 1996;75: 563-73

56. Hammes HP, Brownlee M, Jonczyk A, et al. Subcutaneous injection of a cyclic peptide antagonist of vitronectin receptor type integrins inhibits retinal neovascularization. Nat Med 1996;2:52933.

57. Yasukawa T, Hoffmann S, Eichler W, et al. Inhibition of experimental choroidal neovascularization in rats by an alpha(v)-integrin antagonist. Curr Eye Res 2004;28:359-66.

58. $\mathrm{Fu} \mathrm{Y}$, Ponce ML, Thill $\mathrm{M}$, et al. Angiogenesis inhibition and choroidal neovascularization suppression by sustained delivery of an integrin antagonist, EMD478761. Invest Ophthalmol Vis Sci 2007;48:5184-90.

59. Santulli RJ, Kinney WA, Ghosh S, et al. Studies with an orally bioavailable $\alpha \mathrm{V}$ integrin antagonist in animal models of ocular vasculopathy: retinal neovascularization in mice and retinal vascular permeability in diabetic rats. J Pharmacol Exp Ther 2008;324:894901.

60. Maier A-K B, Kociok N, Zahn G, et al. Modulation of hypoxia-induced neovascularization by JSM6427, an integrin alpha5beta 1 inhibiting molecule. Curr Eye Res 2007;32:801-12.

61. Quiroz-Mercado H. Integrin peptide therapy in choroidal and retinal neovascularization. Retina Today 2013. Available from: http://retinatoday. com/2013/09/integrin-peptide-therapyin-choroidal-and-retinal-neovascularization/

62. Hageman GS, Luthert PJ, Victor Chong $\mathrm{NH}$, et al. An integrated hypothesis that considers drusen as biomarkers of immune-mediated processes at the RPE-Bruch's membrane interface in aging and age-related macular degeneration. Prog Retin Eye Res 2001;20:70532.

63. Anderson DH, Mullins RF, Hageman GS, Johnson LV. A role for local inflammation in the formation of drusen in the aging eye. Am J Ophthalmol 2002;134: 411-31.

64. Anderson DH, Radeke MJ, Gallo NB, et al. The pivotal role of the complement system in aging and age-related macular degeneration: hypothesis re-visited. Prog Retin Eye Res 2010;29:95-112.

65. Olson JL, Courtney RJ, Mandava N. Intravitreal infliximab and choroidal neovascularization in an animal model. Arch Ophthalmol 2007;125:1221-4.

66. Theodossiadis PG, Liarakos VS, Sfikakis PP, et al. Intravitreal administration of the anti-TNF monoclonal antibody infliximab in the rabbit. Graef Arch Clin Exp 2009;247:273-81.

67. Theodossiadis PG, Liarakos VS, Sfikakis PP, et al. Intravitreal administration of the antitumor necrosis factor agent infliximab for neovascular age related macular degeneration. Am J Ophthalmol 2009;147:825-30.

68. Bradley DT, Zipfel PF, Hughes AE. Complement in age-related macular degeneration: a focus on function. Eye 
(Lond) 2011;25:683-93.

69. Kaushal S, Grossi F, Francois C, et al. Complement C3 inhibitor POT-4: clinical safety of intravitreal administration. Invest Ophthalmol Vis Sci 2009;50:5010.

70. Powell JD, Pollizzi KN, Heikamp EB, Horton MR. Regulation of immune responses bymTOR. Annu Rev Immunol 2012;30:39-68.

71. Hay N, Sonenberg N. Upstream and downstream of Mtor. Genes Dev 2004;18:1926-45.

72. Kajiwara M, Masuda S. Role of $\mathrm{mTOR}$ Inhibitors in Kidney Disease. Int J Mol Sci 2016;2016:17.

73. Dejneka NS, Kuroki AM, Fosnot J, et al. Systemic rapamycin inhibits retinal and choroidal neovascularization in mice. Mol Vis 2004;10:964-72.

74. Yagasaki R, Nakahara T, Ushikubo H, et al. Anti-angiogenic effects of mammalian target of rapamycin inhibitors in a mouse model of oxygen-induced retinopathy. Biol Pharm Bull 2014;37:1838-42.

75. Dalal M, Jacobs-El N, Nicholson B, et al. Subconjunctival Palomid 529 in the treatment of neovascular age-related macular degeneration. Graef Arch Clin Exp 2013;251:2705-9.

76. Rittenhouse KD, Johnson TR, Vicini P, et al. RTP801 gene expression is differentially upregulated in retinopathy and is silenced by PF-04523655, a 19-Mer siRNA directed against RTP801. Invest Ophthalmol Vis Sci 2014;55:1232-40.

77. Nguyen QD, Schachar RA, Nduaka CI, et al. PF-04523655 Study Group. Phase 1 dose-escalation study of a siRNA targeting the RTP801 gene in age-related macular degeneration patients. Eye
(Lond) 2012;26:1099-105.

78. Nguyen QD, Schachar RA, Nduaka CI, et al. MONET Clinical Study Group Evaluation of the siRNA PF-04523655 versus ranibizumab for the treatment of neovascular age-related macular degeneration (MONET Study). Ophthalmology 2012;119:1867-73.

79. Nagai N, Klimava A, Lee WH, et al. CTGF is increased in basal deposits and regulates matrix production through the ERK (p42/p44mapk) MAPK and the p38 MAPK signaling pathways. Invest Ophthalmol Vis Sci 2009;50:1903-10.

80. Kothary PC, Badhwar J, Weng C, Del Monte MA. Impaired intracellular signaling may allow up-regulation of CTGF-synthesis and secondary periretinal fibrosis in human retinal pigment epithelial cells from patients with agerelated macular degeneration. Adv Exp Med Biol 2010;664:419-28.

81. Lu F, Hu Z, Sinard J, et al. Factor VIIverteporfin for targeted photodynamic therapy in a rat model of choroidal neovascularization. Invest Ophthalmol Vis Sci 2009;50:3890-6.

82. Nepomuceno RR, Henschen-Edman AH, Burgess WH, Tenner AJ. cDNA cloning and primary structure analysis of $\mathrm{C} 1 \mathrm{qR}(\mathrm{P})$, the human $\mathrm{C} 1 \mathrm{q} / \mathrm{MBL} / \mathrm{SPA}$ receptor that mediates enhanced phagocytosis in vitro. Immunity $1997 ; 6: 119-$ 29.

83. Park M, Tenner AJ. Cell surface expression of $\mathrm{C} 1 \mathrm{qRP} / \mathrm{CD} 93$ is stabilized by $\mathrm{O}-$ glycosylation. J Cell Physiol 2003;196:512-22.

84. Fonseca MI, Carpenter PM, Park M, et al. C1qRp, a myeloid cell receptor in blood, is predominantly expressed on endothelial cells in human tissue. J
Leukocyte Biol 2001;70:793-800.

85. Galvagni F, Nardi F, Maida M, et al. CD93 and dystroglycan cooperation in human endothelial cell adhesion and migration adhesion and migration. Oncotarget 2016;7:10090-103.

86. Kao YC, Jiang SJ, Pan WA, et al. The Epidermal Growth Factor-like domain of CD93 is a potent angiogenic factor. PLoS One 2012;7:51647.

87. Orlandini M, Galvagni F, Bardelli M, et al. The characterization of a novel monoclonal antibody against CD93 unveils a new antiangiogenic target. Oncotarget 2014;5:2750-60.

88. Tosi GM, Caldi E, Parolini B, et al. CD93 as a potential target in neovascular age-related Macular Degeneration. J Cell Physiol 2016;232:1767-73.

89. Tosi GM, Caldi E, Neri G, et al. HTRA1 and TGF- $\beta 1$ concentrations in the aqueous humor of patients with neovascular age-related macular degeneration. Invest Ophthalmol Vis Sci 2017;58:162-7.

90. Yang Z, Camp NJ, Sun H, et al. A variant of the HTRA1 gene increases susceptibility to age-related macular degeneration. Science 2006;314:992-3.

91. Balasubramanian SA, Krishna KK, Baird PN. The role of proteases and inflammatory molecules in triggering neovascular age-related macular degeneration: basic science to clinical relevance. Transl Res 2014;164:179-92.

92. Ciferri C, Lipari MT, Liang WC, et al. The trimeric serine protease HtrA1 forms a cage-like inhibition complex with an anti-HtrA1 antibody. Biochem J 2015;472:169-81. 